\title{
Histerectomia Vaginal Assistida por Laparoscopia em Pacientes com Necessidade de Anexectomia
}

\author{
Laparoscopically Assisted Vaginal Histerectomy in \\ Patients Requiring Adnexectomy
}

\begin{abstract}
Francisco José Cândido dos Reis, Antônio Alberto Nogueira Jurandyr Moreira de Andrade, Hélio Humberto Angotti Carrara Patrícia de Almeida Silva Reis, Sérgio Bighetti
\end{abstract}

\section{RESUM0}

Objetivos: avaliar as vantagens da laparoscopia como instrumento para conversão de histerectomias abdominais em vaginais em pacientes com indicação de anexectomia concomitante, considerando-se a segurança e os custos hospitalares adicionais relativos ao procedimento.

Pacientes e Métodos: estudo de caso controle envolvendo 9 pacientes submetidas à Histerectomia Vaginal Assistida por Laparoscopia (HVAL) e 18 pacientes-controle submetidas à Histerectomia Abdominal (HA), associadas à anexectomia. Foram avaliadas as características pré-operatórias e os resultados do procedimento. Os grupos HVAL e HA são semelhantes quanto à idade, paridade, cesáreas anteriores, cirurgias prévias e IMC.

Resultados: o grupo HVAL apresentou tempo cirúrgico médio de 163, 9 minutos e o grupo HA de 142,8 minutos. Não ocorreram complicações pós-operatórias no grupo HVAL, ao passo que no grupo HA houve 2 casos de deiscência de sutura e 1 caso de hérnia incisional. A mediana do tempo de internação foi de 1 dia no grupo HVAL e 2 dias no grupo HA; a mediana do periodo de convalescença por sua vez foi de 2 e 4 semanas respectivamente. No grupo HVAL, 55,6\% das pacientes necessitaram de medicação analgésica no pós-operatório, o que ocorreu em $100 \%$ das pacientes do grupo HA.

Conclusões: a HVAL mostrou-se vantajosa em relação à HA em termos de melhor recuperação e menor incidência de complicações no pós-operatório. O procedimento é factivel com segurança em um Hospital Universitário, não implicando tampouco em custos adicionais com equipamentos ou instrumental.

PALAVRAS-CHAVE: Histerectomia. Laparoscopia. Custos em saúde.

Departamento de Ginecologia e Obstetrícia da Faculdade de Medicina de Ribeirão Preto - USP Correspondência:

Francisco José Cândido dos Reis

Av. Bandeirantes, $3900-8^{\circ}$ andar

14049-900 - Ribeirão Preto - SP

Tel. (016) 633-0216 - Fax (016) 633-0946
Introdução

A histerectomia é a cirurgia ginecológica mais realizada atualmente, sendo que em até $75 \%$ dos casos a via de acesso escolhida é a abdominal ${ }^{21}$. A 
via vaginal, contudo, parece apresentar várias vantagens em relação à anterior: menos complicações pós-operatórias, melhor recuperação e custos hospitalares reduzidos. A incidência de complicações na histerectomia abdominal pode ser até $70 \%$ maior do que na histerectomia vaginal ${ }^{7}$. Embora apresente menor morbidade, menor tempo cirúrgico, permanência hospitalar reduzida e recuperação mais rápida, a via vaginal é pouco utilizada. Várias situações são apresentadas como contra-indicações para a histerectomia vaginal, destacando-se: suspeita de aderências entre o útero e outras estruturas pélvicas, pouca mobilidade e ausência de descenso do útero, miomas volumosos, suspeita de doença inflamatória pélvica, o conhecimento ou suspeita de massa anexial, necessidade de garantia da remoção dos ovários e falta de habilidade do cirurgião em realizar procedimentos vaginais ${ }^{10}$.

A necessidade de ooforectomia bilateral associada à histerectomia está presente em cerca de $37 \%$ dos casos antes de 45 anos e em $68 \%$ dos casos após os 45 anos. Esta representa, portanto, um importante fator na opção pela laparotomia como via de acesso para a realização de histerectomia, o que é efetivamente verificado independente da idade das pacientes ${ }^{20,21}$.

A laparoscopia é reconhecidamente um excelente método para a abordagem de várias doenças benignas da pelve e em diversas situações oferece vantagens cirúrgicas: possibilita a inspeção da cavidade abdominal, permite a lise de aderências, a mobilização segura e remoção de tumores anexiais benignos e a secção dos ligamentos uterinos redondo e largo, de difícil acesso pela via vaginal. Além disso, no caso de anexectomia planejada a laparoscopia pode ser o procedimento auxiliar para mobilizar seguramente ovários localizados fora da pelve. A confiabilidade da laparoscopia para a abordagem diagnóstica e terapêutica das massas anexiais é reconhecida ${ }^{3}$, representando uma técnica segura e reprodutivel ${ }^{15}$.

A laparoscopia pode representar um passo importante na eliminação de algumas contraindicações para a realização da histerectomia vaginal. A ampliação de seu uso poderia reduzir as indicações de procedimentos abdominais em favor da via vaginal ${ }^{11}$. Em que pesem os benefícios representados pela laparoscopia na conversão de procedimentos de indicação inicial por via abdominal para a via vaginal, ainda restam dúvidas quanto à sua reprodutibilidade técnica tendo em vista possiveis custos mais elevados de equipamentos e necessidade de longos períodos de treinamento.

O objetivo deste trabalho é investigar a possibilidade de conversão segura, mediante laparoscopia, de histerectomias abdominais em vaginais nas pacientes com indicação de anexectomia concomitante, as implicações clínicas desta nova técnica e a necessidade de equipamentos adicionais àqueles utilizados rotineiramente por um serviço de endoscopia ginecológica, ou seja, a ocorrência de impacto significativo nos custos da intervenção.

\section{Pacientes e Métodos}

\section{Casuistica}

No periodo de julho de 1997 a outubro de 1998, 9 pacientes com indicação de histerectomia total e anexectomia foram selecionadas para a realização da Histerectomia Vaginal Assistida por Laparoscopia (HVAL). Os resultados foram avaliados em relação a um grupo controle constituído por uma série histórica de 18 pacientes submetidas à Histerectomia Abdominal (HA) associada a anexectomia no período de dezembro de 1995 a novembro de 1996 . Todas as pacientes, inclusive as do grupo controle, foram atendidas no Serviço de Oncologia Tocoginecológica do Hospital das Clínicas de Ribeirão Preto da Faculdade de Medicina de Ribeirão Preto da USP, sendo as indicações sempre por consenso no grupo, conforme apresentado na Tabela 1.

Tabela 1 - Indicações e diagnóstico final das pacientes submetidas a HVAL.

\begin{tabular}{|c|c|c|}
\hline Caso & Indicação & Diagnóstico final \\
\hline 1 & Hiperplasia endometrial & $\begin{array}{l}\text { Hiperplasia endometrial com atipia acentuada e } \\
\text { hiperplasia de estroma ovariano }\end{array}$ \\
\hline 2 & $\begin{array}{l}\text { Aumento do volume ovariano e história } \\
\text { familiar de câncer }\end{array}$ & Cistos de inclusão peritoneal no ovário esquerdo \\
\hline 3 & $\begin{array}{l}\text { Carcinoma "in situ" de colo uterino e } \\
\text { massa anexial }\end{array}$ & NIC III e hidrossalpinge bialateral \\
\hline 4 & Massa anexial & Cistadenoma mucinoso \\
\hline 5 & Carcinoma "in situ" de colo uterino & $\mathrm{Ca}$ "in situ" de colo uterino \\
\hline 6 & $\begin{array}{l}\text { Massa anexial, pólipo endometrial, NIC } \\
\text { III }\end{array}$ & $\begin{array}{l}\text { Cervicite crônica, pólipo endometrial hiperplásico e } \\
\text { fibroma de ovário direito }\end{array}$ \\
\hline 7 & Massa anexial & Cistadenocarcinoma "borderline" de ovário direito (Ia) \\
\hline 8 & Massa anexial & Cistadenoma seroso de ovário esquerdo \\
\hline 9 & Massa anexial & Cistadenoma seroso de ovário direito \\
\hline
\end{tabular}

Obs: Todas as pacientes do grupo controle tiveram como diagnóstico inicial massa anexial e todos os diagnósticos finais foram de doença benigna.

\section{Metodologia}

Na elaboração da hipótese diagnóstica préoperatória foram utilizados a avaliação clínica, 
métodos laboratoriais e de imagem conforme os protocolos vigentes no serviço. Todas as pacientes foram submetidas a uma avaliação de risco cirúrgico e os problemas clínicos intercorrentes corrigidos quando necessário. O preparo préoperatório consistiu de jejum de no mínimo 10 horas, lavagem intestinal com água e glicerina na véspera e administração de antibióticos profiláticos.

Todas as HVAL foram realizadas em centro cirúrgico, sob anestesia geral e pelo mesmo cirurgião, com a paciente colocada em posição de litotomia dorsal e pernas semi fletidas a $30^{\circ}$. Utilizou-se um equipamento Storz, com ótica de $10 \mathrm{~mm}$ de visão frontal $\left(0^{\circ}\right)$, fonte de luz Xenônio e insuflador automático de $\mathrm{CO}_{2}$ com fluxo de 2,5 a 3,0 litros por minuto e pressão máxima entre $12 \mathrm{e}$ $15 \mathrm{mmHg}$.

O procedimento iniciou-se pelo preparo do campo operatório, esvaziamento vesical e adaptação do manipulador uterino por via vaginal. Procedeu-se então a incisão inicial intra-umbilical inferior e punção com agulha de Verres para distensão da cavidade peritoneal com $\mathrm{CO}_{2}$. Foram então inseridos um trocarte de $10 \mathrm{~mm}$ na incisão umbilical e dois trocartes de $5 \mathrm{~mm}$ laterais ao músculo reto abdominal entre 3 e $5 \mathrm{~cm}$ acima da sínfise púbica, estes últimos sob visualização direta. Inicialmente, foram inspecionados o intestino delgado, o apêndice, o figado, a vesícula biliar, a superfície diafragmática, o peritôneo parietal e as demais estruturas do abdome superior. Os ureteres foram identificados na borda da pelve e seguidos até junto ao cruzamento com as artérias uterinas. Procedeu-se então a inspeção detalhada da pelve e a realização de lavados peritoneais para citologia.

A HVAL teve início pela cauterização e secção dos ligamentos redondos. A bexiga foi dissecada do segmento uterino e do colo até a cúpula vaginal anterior com o uso de tesoura endoscópica. Os infundíbulos ovarianos foram dissecados, cauterizados com bisturi bipolar, seccionados e ligados com fio de vicryl 0 . Os ligamentos largos foram seccionado e os vasos do pedículo uterino expostos. Os ligamentos uterossacros foram cauterizados e seccionados, iniciando-se então o tempo vaginal.

O procedimento vaginal consistiu da apreensão do colo seguida de uma incisão circular na cúpula vaginal. Os ligamentos cardinais foram identificados, ligados e seccionados, assim como eventuais porções remanescente dos ligamentos uterossacros. Os vasos uterinos foram facilmente ligados e o útero em conjunto com os anexos foram retirados pela incisão vaginal. A porção central do peritôneo pélvico foi suturada por via vaginal. Os ligamentos cardinais e uterossacros foram utilizados para a pexia da cúpula vaginal, a qual foi suturada com pontos separados.

Todos os tumores anexiais foram submetidos a exame anátomo-patológico de congelação transoperatório.

A análise estatística dos resultados foi realizada com auxílio do software GraphPad Prism 2.01. Utilizou-se o teste t de Student não-pareado para a análise das variáveis quantitativas contínuas de distribuição normal, o teste de Mann-Whitney para a análise das variáveis quantitativas de distribuição livre (não-paramétrica) e o teste exato de Fisher para a análise das tabelas de contingência.

\section{Validação do grupo controle}

A caracterização pré-operatória das pacientes submetidas à Histerectomia Vaginal Assistida por Laparoscopia (HVAL) e à Histerectomia Abdominal (HA) é apresentada na Tabela 2. As pacientes do grupo HVAL apresentaram média de idade igual a 52,67 anos (40 a 62) e as do grupo HA igual a 54,33 anos (36 a 78), não sendo esta diferença estatisticamente significante (teste t não-pareado; $p=0,71$ ).

Tabela 2 - Características das pacientes submetidas à Histerectomia Vaginal Assistida por Laparoscopia (HVAL) e das pacientes submetidas à Histerectomia Abdominal (HA).

\begin{tabular}{lccc}
\hline Característica & HVAL & HA & p \\
\hline Idade (anos) & $52,67(40$ a 62$)$ & $54,33(36$ a 78$)$ & 0,71 \\
Paridade & $3(0$ a 10$)$ & $4(0$ a 14$)$ & 0,78 \\
Proporção de & 33,3 & 22,2 & 0,65 \\
cesáreas & & & \\
Cirurgia prévia & $44,4 \%$ & $27,8 \%$ & 0,42 \\
I M C & $28,37(23,69$ a 38,32$)$ & $27,16(20,31$ a 37,58$)$ & 0,54 \\
\end{tabular}

Quanto à paridade, o grupo de pacientes submetidas a HVAL apresentou mediana de 3 partos $(0$ - 10) e o grupo HA mediana de 4 partos (0 - 14), não sendo esta diferença estatisticamente significante (teste de Mann-Whitney; $p=0,78$ ) O número de pacientes com cesárea prévia foi 3 no grupo HVAL e 4 no grupo HA, não sendo a diferença estatisticamente significante (teste exato de Fisher, $\mathrm{p}=0,65)$. No grupo de pacientes submetidas à HVAL, 4/9 apresentavam cirurgia abdominal prévia e no grupo de pacientes submetidas à $\mathrm{HA} 5 / 13$, não havendo diferença estatisticamente significante (teste exato de Fisher, $p=0,42$ ). A média do Índice 
de Massa Corporal (IMC) das pacientes submetidas à HVAL foi $28,37(23,69$ a 38,32$)$ e das pacientes submetidas à HA de 27,16 (20,31 a 37,58), não sendo também observada diferença estatisticamente significante (teste t não-pareado; $\mathrm{p}=0,54)$.

\section{Resultados}

Os resultados da conversão para via vaginal de histerectomias de indicação inicial pela via abdominal estão apresentados na Tabela 3.

Tabela 3 - Comparação entre os grupos de pacientes submetidas à HVAL e à HA, segundo resultados intra-operatórios e pós-operatórios.

\begin{tabular}{lccc}
\hline Parâmetro & HVAL & HA & p \\
Duração da cirurgia (min) & $163,9(100$ a 240$)$ & $142,8(100$ a 195) & 0,13 \\
Uso de analgésicos & $55,60 \%$ & $100 \%$ & 0,007 \\
Complicações PO & 0 & 3 & 0,53 \\
Internação PO (dias) & 1 (1 a 2$)$ & $2(1$ a 11$)$ & 0,03 \\
Atividades habituais (semanas) & $2(1$ a 4$)$ & $4(2$ a 8$)$ & 0,0003
\end{tabular}

A média do tempo cirúrgico foi de 163,9 minutos (100 a 240) no grupo HVAL e de 142,8 (100 a 195) no grupo HA (teste t não-pareado $p=0,13$ ) (Gráfico 1). Não houve complicações intraoperatórias em nenhum dos dois grupos. Na avaliação da dor pós-operatória, $5 / 9$ das pacientes submetidas a HVAL e 18/18 das pacientes submetidas a HA solicitaram medicação analgésica após o término dos efeitos anestésicos, sendo esta diferença estatisticamente significante (teste exato de Fisher, $p=0,007)$. As pacientes do grupo HVAL não apresentaram complicações pós-operatórias, ao passo que no grupo HA ocorreram dois casos de deiscência e 1 caso de hérnia incisional. A mediana do tempo de internação pós-operatória nas pacientes submetidas à HVAL foi de 1 dia ( 1 a 2 ) e nas pacientes submetidas a HA de 2 dias ( 1 a 11 ), diferença estatisticamente significante (teste de Mann-Whitney, $p=0,03)$. O retorno às atividades habituais, estimado em semanas, foi mais precoce no grupo HVAL, com mediana de 2 semanas (1 a 4) do que no grupo HA, com mediana de 4 semanas (2 a 8); esta diferença foi estatisticamente significante (teste de Mann-Whitney, $p=0,0003$ ).

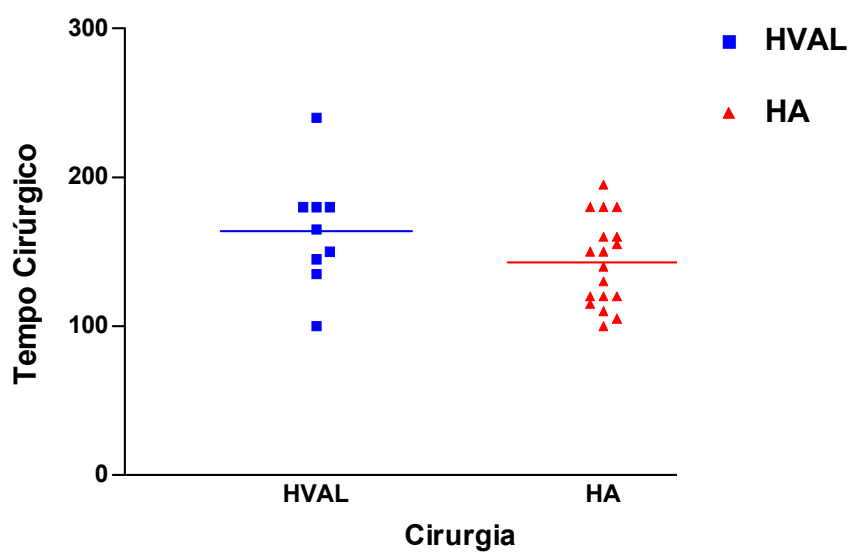

Gráfico 1 - Distribuição das pacientes segundo o tempo cirúrgico e o tipo de cirurgia.

\section{Discussão}

A extensão da incisão tem um profundo efeito na incidência de complicações pós-operatória em cirurgias abdominais, tanto imediatas quanto a médio prazo ${ }^{12}$. A escolha da via de acesso constitui, portanto, um importante fator para o sucesso no tratamento cirúrgico das doenças uterinas e anexiais. Quando se escolhe o tipo de incisão abdominal para a realização de uma histerectomia, deve-se considerar a exposição operatória necessária, a presença de incisões abdominais prévias, o tamanho e a integridade da cicatriz resultante, além da aparência estética.

Várias publicações apresentam resultados melhores quando se associa a laparoscopia para a realização de histerectomias: menos dor e menor necessidade de analgésicos no pós-operatório, permanência hospitalar e tempo de convalescença reduzidos, além de menor incidência de complicações $^{2,13}$. Contudo, tais resultados são questionados pela heterogeneidade das indicações e pela falta de um grupo controle adequado. As características pré-operatórias observadas na Tabela 2, assim como a homogeneidade das indicações, ou seja, histerectomia associada a anexectomia em ambos os grupos, HVAL e HA, permitem concluir pela adequação do grupo controle e validade dos resultados obtidos.

O tempo operatório no grupo HVAL apresentou uma tendência a ser superior ao do grupo HA, conforme observado no Gráfico 1. Em séries maiores observa-se um declínio progressivo no tempo operatório conforme aumenta a experiência da equipe cirúrgica. Entretanto é 
importante ressaltar que o tempo cirúrgico por si só não reflete qualidade superior de cirurgia. O tempo cirúrgico depende de uma série de variáveis como: habilidade do operador e seus auxiliares, indicação da histerectomia, dificuldades de acesso relacionados com aderências, miomas grandes ou obesidade, além do desempenho do equipamento e do instrumental utilizado, o que de certa forma explica a grande variação encontrada na literatura ${ }^{17}$.

A HVAL oferece vantagens tanto sobre a via abdominal quanto sobre a via estritamente vaginal. A laparoscopia permite a visualização dos ureteres e previne lesões inadvertidas, assim como para a anexectomia concomitante, os vasos ovarianos são ligados sob visão direta assegurando hemostasia adequada. Embora cirurgiões muito experientes em cirurgia vaginal possam realizar com sucesso a histerectomia total com anexectomia pela via vaginal $^{1,22}$, os resultados de estudos multicêntricos demonstram que em mais de $75 \%$ dos casos a via abdominal é a eleição $0^{20,21}$. Os excelentes resultados publicados por Sheth ${ }^{19}$, que afirma ser possivel a anexectomia com histerectomia vaginal em 95\% de casos, não permitem considerar que esta operação seja realmente possível em 95\% casos ou ainda que seja um procedimento simples. O cirurgião em questão é muito experiente em cirurgia vaginal, como também os pacientes de sua série são cuidadosamente selecionados ${ }^{6}$.

Em nosso estudo, a associação da laparoscopia e conseqüente utilização da via vaginal mostrou diversos benefícios em comparação com a via abdominal: menor incidência de complicações pós-operatórias, menor uso de analgésicos, ou seja menos dor pós-operatória, permanência hospitalar reduzida e retorno precoce às atividades habituais, confirmando os achados da literatura ${ }^{16}$.

Embora a viabilidade de se realizar histerectomia total por laparoscopia esteja bem demonstrada, ainda há dúvidas quanto às indicações para histerectomia ${ }^{4,9,21}$ e quando bem estabelecidas, quanto às indicações para a abordagem laparoscópica ${ }^{6,8}$. A cirurgia laparoscópica não representa uma alternativa para a cirurgia vaginal que possa ser originalmente realizada sob boas condições ${ }^{18}$. A HVAL só deve ser proposta como um meio de facilitar uma histerectomia vaginal evitando-se assim a laparotomia ${ }^{5}$. Levando se em conta tanto aspectos individuais quanto sociais, são consideráveis os beneficios quando se evita a laparotomia.

A existência de doença anexial é uma indicação para o uso da laparoscopia, desde que nenhum sinal de malignidade tenha sido observado durante a investigação pré-operatória. Em uma população de pacientes sem prolapso uterino ou relaxamento do assoalho pélvico, esta abordagem permitiu reduzir a $12,5 \%$ a taxa de laparotomias para a realização de histerectomia associada à anexectomia 6 .

Observamos ainda que é perfeitamente possivel realizar a HVAL com um conjunto simples, barato e reutilizável de instrumentos, empregado rotineiramente no Setor de Endoscopia Ginecológica. Este ponto é de grande importância, lembrando que um dos argumentos principais contra a HVAL tem sido o custo superior ao de laparotomia. Se A HVAL é realizada com equipamento exclusivamente reutilizável, o custo hospitalar da operação passa a ser comparável ao da histerectomia vaginal ou da abdominal ${ }^{14}$.

Além das vantagens em termos de redução de complicações e sequelas pós-operatórias, a introdução da histerectomia vaginal assistida por laparoscopia em um serviço universitário de treinamento de médicos residentes pode levar ao aumento do número de casos de procedimentos vaginais com maior aprendizado da técnica vaginal.

\section{SUMMARY}

Purpose: to evaluate the advantages of the laparoscopic approach for conversion of abdominal hysterectomies in vaginal hysterectomies in patients with indication of concomitant adnexectomy, being considered the safety and the additional costs of the procedure.

Patients and Methods: cases: 9 patients submitted to Laparoscopically Assisted Vaginal Hysterectomy (LAVH) associated with adnexectomy. Controls: 18 patients submitted to Abdominal Hysterectomy (AH) associated with adnexectomy. Both groups were compared regarding preoperative characteristics and the results of the procedure. The patients submitted to LAVH and AH are similar concerning age, parity, cesarean deliveries, previous surgeries and body mass index.

Results: the average surgery time was 163.9 minutes for patients submitted to LAVH and 142.8 minutes for patients submitted to AH. No patient in the LAVH group presented postoperative complications, while in the AH group 2 patients presented suture deiscence and there was 1 case of incisional hernia. The median of hospital stay was 1 day in the LAVH group and 2 days in the AH group, those of convalescence periods were 2 and 4 weeks, respectively. 55.6\% of the patients in the LAVH group and $100 \%$ in the AH group needed analgesics in the postoperative period.

Conclusions: LAVH was shown to be advantageous in relation to AH in terms of better recovery and lower incidence of complications in the postoperative period. The procedure is feasible and safe in a University Hospital, and without additional costs.

KEY WORDS: Hysterectomy. Laparoscopy. Health costs. 
Referências

1. Ballard LA, Walters MD. Transvaginal mobilization and removal of ovaries and fallopian tubes after vaginal hysterectomy. Obstet Gynecol 1996 87: 35-9.

2. Boike GM, Elfstrand EP, DelPriore G, Schumock D, Holley HS, Lurain JR. Laparoscopically assisted vaginal hysterectomy in a university hospital: report of 82 cases and comparison with abdominal and vaginal hysterectomy. Am J Obstet Gynecol 1993; 168: 1690-7.

3. Canis M, Mage G, Pouly JL, Wattiez A, Manhes H, Bruhat MA. Laparoscopic diagnosis of adnexal cystic masses: a 12-year experience with long-term follow-up. Obstet Gynecol 1994; 83: 707-12.

4. Carlson KJ, Nichols DH, Schiff I. Indications for hysterectomy. N Engl J Med 1993; 25: 856-60.

5. Chapron C, Dubuisson JB. Laparoscopic hysterectomy [letter; comment]. Lancet 1995; 345:8949.

6. Chapron C, Dubuisson JB, Ansquer Y, Capella Allouc S. Hysterectomy with adnexectomy. Can operative laparoscopy offer advantages? J Reprod Med 1997; 42: 201-6.

7. Dicker RC, Greenspan JR, Strauss LT, Cowart MR, Scally MJ, Peterson HB et al. Complications of abdominal and vaginal hysterectomy among women of reproductive age in the United States. The Collaborative Review of Sterilization. Am J Obstet Gynecol 1982; 144:7, 841-8.

8. Dorsey JH, Steinberg EP, Holtz PM. Clinical indications for hysterectomy route: patient characteristics or physician preference?. Am J Obstet Gynecol 1995; 173: 1452-60.

9. Finkel ML, Finkel DJ. The effect of a second opinion program on hysterectomy performance. Med Care 1990; 28: 776-83.

10.Fylstra DL, Carter JF. Laparoscopically assisted vaginal hysterectomy in a university hospital.
Decreasing the need for abdominal hysterectomy and increasing resident experience in vaginal surgery. J Reprod Med 1996; 41:7, 497-503.

11.Harris MB, Olive DL. Changing hysterectomy patterns after introduction of laparoscopically assisted vaginal hysterectomy. Am J Obstet Gynecol 1994; 171: 340-3.

12.Ledet WP. Ambulatory cholecystectomy without disability. Arch Surg 1990; 125: 1434-5.

13. Minelli L, Angiolillo M, Caione C, Palmara V. Laparoscopically-assisted vaginal hysterectomy. Endoscopy 1991; 23:2, 64-6.

14.Nezhat C, Bess O, Admon D, Nezhat CH, Nezhat F. Hospital cost comparison between abdominal, vaginal, and laparoscopy-assisted vaginal hysterectomies. Obstet Gynecol 1994; 83: 713-6.

15.Nezhat F, Nezhat C, Silfen SL. Videolaseroscopy for oophorectomy. Am J Obstet Gynecol 1991; 165: 1323-30.

16.Olsson JH, Ellstrom M, Hahlin M. A randomised prospective trial comparing laparoscopic and abdominal hysterectomy. Br J Obstet Gynaecol 1996; 103: 345-50.

17.Rempen A. Laparoscopic assistance at vaginal hysterectomy: a literature review. Arch Gynecol Obstet 1996; 258: 57-64.

18.Richardson RE, Bournas N, Magos AL. Is laparoscopic hysterectomy a waste of time? Lancet 1995 ; 345: 36-41.

19.Sheth SS. The place of oophorectomy at vaginal hysterectomy. Br J Obstet Gynaecol 1991; 98: 662-6.

20.Vessey MP, Villard-Mackintosh L, McPherson K, Coulter A, Yeates D. The epidemiology of hysterectomy: findings in a large cohort study. Br J Obstet Gynaecol 1992; 99:5, 402-7.

21.Wilcox LS, Koonin LM, Pokras R, Strauss LT, Xia Z, Peterson HB. Hysterectomy in the United States, 1988-1990. Obstet Gynecol 1994; 83:4, 549-55.

22.Wright RC. Vaginal oophorectomy. Am J Obstet Gynecol 1974; 120: 759-63.

\begin{tabular}{|c|}
\hline COMUNICADO AOS ASSOCIADOS E LEITORES \\
Para informações e consultas temos à disposição quatro endereços eletrônicos: \\
febrasgopresidencia@alphanet.com.br \\
secretaria_executiva@febrasgo.org.br \\
publicacoes@febrasgo.org.br \\
tego_habilitacao@febrasgo.org.br
\end{tabular}

